\title{
SOME REMARKS ON THE STEFAN PROBLEM WITH SURFACE STRUCTURE
}

\author{
BY \\ MORTON E. GURTIN AND H. METE SONER \\ Carnegie-Mellon University, Pittsburgh, Pennsylvania
}

\begin{abstract}
This paper discusses a generalized Stefan problem which allows for supercooling and superheating and for capillarity in the interface between phases. Simple solutions are obtained indicating the chief differences between this problem and the classical Stefan problem. A weak formulation of the general problem is given.
\end{abstract}

1. Introduction. In this paper we discuss a generalized Stefan problem which allows for capillarity in the interface between phases. We use the following notation: $\Omega$ is a region in $\mathbb{R}^{d}$ occupied by a two-phase continuum; $\Omega_{i}(t)$ is the subregion of $\Omega$ occupied by phase $i(i=1,2) ; \jmath(t)$ is the interface ${ }^{1}$ between $\Omega_{1}(t)$ and $\Omega_{2}(t)$; $\mathbf{n}(x, t)$ is the unit normal to $s(t)$ directed outward from $\Omega_{1}(t) ; v(x, t)$ is the normal velocity of $s(t)$ in the direction of $\mathbf{n}(x, t)$;

$$
\mathbb{L}(x, t)=-\nabla_{s} \mathbf{n}(x, t) \in \operatorname{lin}\left(\mathbf{n}(x, t)^{\perp}\right)
$$

is the curvature tensor and $\kappa(x, t)=(d-1)^{-1}$ trace $\mathbb{L}(x, t)$ the mean curvature of $s(t)$; and

$$
\chi(x, t)= \begin{cases}0 & \text { if } x \in \Omega_{1}(t), \\ 1 & \text { if } x \in \Omega_{2}(t),\end{cases}
$$

is the phase distribution. Here $\operatorname{lin}(A)$ is the set of linear transformations of $A$ into itself, while $\nabla_{s}$ is the surface gradient ${ }^{2}$ on $s(t)$.

The problem under consideration consists of: the partial differential equations

$$
\begin{array}{ll}
c_{1} u_{t}=-\operatorname{div} \mathbf{q}, & \mathbf{q}=-\mathbf{K}_{1} \nabla u \text { in phase } 1, \\
c_{2} u_{t}=-\operatorname{div} \mathbf{q}, & \mathbf{q}=-\mathbf{K}_{2} \nabla u \text { in phase } 2 ;
\end{array}
$$

the free-boundary conditions ${ }^{3}$

$$
u=\mathbb{B}(\mathbf{n}) \cdot \mathbb{L}-\beta(\mathbf{n}) v, \quad l v=[\mathbf{q}] \cdot \mathbf{n} \quad \text { on the interface; }
$$

Received October 24, 1990.

${ }^{1}$ To avoid discussions of contact conditions, we assume throughout that the interface does not touch the container walls: $\lrcorner(t) \cap \partial \Omega=\varnothing$.

${ }^{2} \nabla$ and div will denote the gradient and divergence in $\mathbb{R}^{d}$. The subscripts $t$ and $r$ denote partial derivatives.

${ }^{3}$ For linear transformations $\mathbf{A}, \mathbf{B}$ of an inner-product space into itself, we write $\mathbf{A} \cdot \mathbf{B}=\operatorname{trace}\left(\mathbf{A} \mathbf{B}^{\top}\right)$ with $\mathbf{B}^{\top}$ the transpose of $\mathbf{B}$. 
the initial conditions

$$
u(x, 0)=u_{0}(x), \quad \chi(x, 0)=\chi_{0}(x)
$$

for $x \in \Omega$; and suitable boundary conditions and/or conditions at infinity. Here "in phase $i$ " means in $\Omega_{i}(t)$ for $t \geq 0$, while "on the interface" means on $\lrcorner(t)$ for $t \geq 0$. Modulo appropriate scalings, $u$ is the temperature; $\mathbf{q}$ is the heat flux; [q] is the jump in $\mathbf{q}$ across the interface (phase 2 minus phase 1 ); $c_{i}$ and $\mathbf{K}_{i} \in \operatorname{lin}\left(\mathbb{R}^{3}\right)$ are the specific heat and conductivity tensor for phase $i(i=1,2) ; l$ is the latent heat; $\mathbb{B}(\mathbf{n}) \in \operatorname{lin}\left(\mathbf{n}^{\perp}\right)$ is the capillarity tensor; $\beta(\mathbf{n})$ is the kinetic coefficient; $u_{0}$ is the initial temperature-distribution; and $\chi_{0}$ is the initial phase-distribution.

In the classical Stefan problem the temperature is strictly negative in the solid and strictly positive in the liquid, and is generally used to characterize the individual phase regions. Here we allow positive temperatures in the solid (superheating), negative temperatures in the liquid (supercooling), and therefore use the phase distribution to characterize the individual regions.

We assume that

$$
l>0
$$

which is consistent with

$$
\text { phase } 1=\text { solid, } \quad \text { phase } 2 \text { = liquid. }
$$

When the two phases and the interface are isotropic, (1.2) and (1.3) have the simple forms

$$
\begin{array}{ll}
c_{1} u_{t}=-\operatorname{div} \mathbf{q}, & \mathbf{q}=-k_{1} \nabla u \text { in the solid, } \\
c_{2} u_{t}=-\operatorname{div} \mathbf{q}, & \mathbf{q}=-k_{2} \nabla u \text { in the liquid, }
\end{array}
$$

and

$$
u=\alpha \kappa-\beta v, \quad l v=[\mathbf{q}] \cdot \mathbf{n} \quad \text { on the interface, }
$$

with $K_{i}, \alpha, \beta$ scalar constants.

Important special cases of the general problem are based on the following assumptions:

(i) $c_{1}=c_{2}=0$ (fast-diffusion);

(ii) $c_{1}=0, \mathbf{K}_{1}=\mathbf{0}$ (one active phase);

(iii) $\beta(\mathbf{n})=0$ (no surface kinetics);

(iv) $\mathbb{B}(\mathbf{n})=\mathbb{D}$ (no capillarity);

(v) $c_{1}=c_{2}=\kappa_{1}=\kappa_{2}=l=0$ (no bulk structure);

with the initial condition for $u$ in phase $i$ omitted when $c_{i}=0$. The isotropic problem without surface kinetics and without capillarity is the classical Stefan problem; the isotropic one-phase problem with capillarity, but without surface kinetics and under the assumption of fast diffusion, ${ }^{4}$ was introduced by Mullins [3] to explain the formation of grooves on an interface separating a solid phase from a saturated ${ }^{5}$

\footnotetext{
${ }^{4}$ These equations also describe the motion of an interface separating immiscible viscous fluids, when the fluids lie in the narrow gap between parallel plates (Hele-Shae cell) (cf. Saffman and Taylor [2] and McLean and Saffman [5]).

${ }^{5}$ In the papers by Mullins [3] and Yokoyama and Kuroda [18] the underlying transport mechanism is mass diffusion rather than heat conduction.
} 
fluid phase; the isotropic problem with two active phases but without surface kinetics was introduced by Mullins and Sekerka [4] to study the stability of crystal growth; the anisotropic one-phase problem with fast diffusion and no capillarity was introduced by Yokoyama and Kuroda [18] to study pattern information in the growth of snow crystals; the isotropic ${ }^{6}$ problem with no bulk structure and $u=0$ was introduced by Mullins [1] to study the motion of grain boundaries; the general problem was derived within a continuum-thermodynamical framework by Gurtin [10].

In this paper we consider the equations described above in conjunction with the boundary conditions

$$
u=U \text { on a portion of } \partial \Omega, \quad \mathbf{q} \cdot \boldsymbol{\nu}=0 \text { on the remainder of } \partial \Omega,
$$

with $U$ constant and $\nu$ normal to $\partial \Omega$, so that a portion of the boundary is isothermal, the remainder insulated. In addition, we allow $\Omega$ to be unbounded, but only for the special case in which

$$
u(x, t) \rightarrow U \text { as }|x| \rightarrow \infty
$$

in a sufficiently regular manner.

In Sec. 2 we discuss an energy equation associated with the problems described above. We show that, because of the instabilities associated with supercooling and superheating, it is plausible to expect solutions in which one phase grows from a seed of zero volume.

In Sec. 3 we discuss the spherically symmetric problem, without surface kinetics, under the assumption of fast diffusion, with the liquid supercooled at infinity. We consider some simple problems that indicate the chief differences between the problems studied here and the classical Stefan problem. In particular, we show that: ${ }^{7}$

(i) for $\Omega=\mathbb{R}^{3}$, a ball of the solid phase of sufficiently small size disappears in finite time, but a sufficiently large ball grows without bound;

(ii) for $\Omega=\mathbb{R}^{3}$ and the solid phase initially situated in a spherical shell of thickness $\varepsilon$, the thickness of the solid shell initially increases, but the inner radius of this region decreases to zero in finite time $T$; the solid ball remaining at time $T$ disappears at a later time or grows without bound according as $\varepsilon$ is less than or greater than a critical value; in the limit $\varepsilon \rightarrow 0$ the region occupied by the solid disappears infinitely fast; the problem has no solution for $\varepsilon=0 ;{ }^{8}$

(iii) when $\Omega$ is the region exterior to a sphere of radius $R$, with the boundary $r=R$ insulated and with the solid phase initially in a spherical shell of zero thickness at $r=R$, the solid phase grows without bound provided $R$ is sufficiently large.

\footnotetext{
${ }^{6}$ The anisotropic problem without bulk structure is discussed by Angenent and Gurtin [12].

${ }^{7}$ While (ii) and (iii) are probably of little practical interest, they do demonstrate-within a manageable geometry - the possibility of growth from a seed of zero volume.

${ }^{8}$ The one-dimensional theory of a flat interface with and without fast diffusion, but without surface kinetics, is discussed by Gurtin, Kossioris, and Soner [15]. There, because there is supercooling but no capillarity, there are solutions in which the solid grows from a set of zero one-dimensional measure. Here the curvature of the interface brings capillary forces into play, and the solid region must have nonzero volume to nucleate.
} 
In Sec. 4 we use recent ideas ${ }^{9}$ of Sethian [9] and Osher and Sethian [11] to note a weak formulation of the system (1.2), (1.3).

2. The free-energy inequality. For a large class of problems of physical interest the capillarity tensor $\mathbb{B}(\mathbf{n})$ is derivable from an interfacial free-energy $f(n) \geq 0$ in the sense that (cf. [10])

$$
\mathbb{B}(\mathbf{n})=f(\mathbf{n}) \mathbb{1}(\mathbf{n})+D^{2} f(\mathbf{n}),
$$

where $\mathbb{1}(\mathbf{n}), D^{2} f(\mathbf{n}) \in \operatorname{lin}\left(\mathbf{n}^{\perp}\right)$ with $\mathbb{1}(\mathbf{n})$ the identity on $\mathbf{n}^{\perp}$ and $D^{2} f(\mathbf{n})$ the second derivative of $f(\mathbf{n})$ on the surface of the unit ball. In this section we assume that such a free-energy exists. We assume in addition that both conductivity tensors are positive definite, while $\beta(\mathbf{n}) \geq 0$.

For convenience, we introduce functions $c(x, t)$ and $\mathbf{K}(x, t)$ defined by

$$
c=l^{-1} c_{2} \chi+l^{-1} c_{1}(1-\chi), \quad \mathbf{K}=l^{-1} \mathbf{K}_{2} \chi+l^{-1} \mathbf{K}_{1}(1-\chi),
$$

and write

$$
\begin{gathered}
\text { vol }=d \text {-dimensional Lebesque measure in } \mathbb{R}^{d}, \\
\text { area }=(d-1) \text {-dimensional Hausdorff measure on surfaces in } \mathbb{R}^{d} .
\end{gathered}
$$

Given any temperature field $u(x)$ and any phase distribution $\chi(x)$, we define the free-energy $\varepsilon\{u, \chi\}$ by

$$
\varepsilon\{u, \chi\}=\int_{0} f(\mathbf{n})+U \operatorname{vol}\left(\Omega_{1}\right)+\frac{1}{2} \int_{\Omega} c(u-U)^{2} .
$$

Then any sufficiently regular solution $u(x, t), \chi(x, t)$ of (1.2), (1.3), (1.8), and (1.9) (for $\Omega$ unbounded) satisfies the free-energy inequality (cf. [10, Eq. (7.9)]):

$$
\frac{d}{d t} \varepsilon\{u, \chi\}+\frac{1}{2}\left(c_{2}-c_{1}\right) \int_{s}(u-U)^{2} v=-\int_{\Omega} \nabla u \cdot \mathbf{K} \nabla u-\int_{s} \beta(\mathbf{n}) v^{2} \leq 0 .
$$

The right side of this equation represents energy dissipated by heat conduction and in the exchange of atoms between phases.

We remark, for future use, that (2.4) is (at least formally) valid when any of $c_{1}, c_{2}, f(\mathbf{n})$, and $\beta(\mathbf{n})$ vanish. Also, when the material is isotropic, $f(\mathbf{n})=f=$ $\alpha /(d-1)$ and

$$
\int_{s} f(\mathbf{n})=f \operatorname{area}(s)
$$

By (2.4), if $c_{1}=c_{2}$ (which holds trivially in the case of fast diffusion), then the free-energy (2.3) is nonincreasing.

Supercooled liquids are inherently unstable; as we shall see, this instability allows for the spontaneous formation of the solid ${ }^{10}$ phase from a seed of zero volume. To study this phenomenon, we consider problems with $\Omega$ initially almost all liquid in the sense that

$$
\chi(x, 0)=1 \text { for a.e } x \in \Omega .
$$

\footnotetext{
${ }^{9}$ Cf. also Barles [8], Chen, Giga, and Goto [13], Evans and Spruck [14], and Soner [17].

${ }^{10}$ The problem for a liquid seed is strictly analogous: in the ensuing discussions simply replace the word solid by liquid, the boundary temperature $U$ by $-U$, and the word supercooled by superheated.
} 
Assume that the diffusion is fast, so that $c_{1}=c_{2}=0$. Then, as an immediate consequence of the free-energy inequality (2.4), a solid seed cannot grow when $U>0$, but such growth is plausible when $U<0$. In this latter case, when the boundary of the liquid phase intersects the portion of the boundary on which $u=U$, at least some of the liquid must be supercooled; thus, in general, supercooling of the liquid is at least formally necessary for the spontaneous growth of a solid seed from a set of zero volume.

Thus suppose that

$$
U<0 \text {. }
$$

Then the liquid equilibrium

$$
u(x, t) \equiv U, \quad \chi(x, t) \equiv 1
$$

is a solution of the basic equations and boundary conditions, but, since $U<0$, the solid equilibrium

$$
u(x, t) \equiv U, \quad \chi(x, t) \equiv 0
$$

has lower free-energy (2.3): the presence of supercooling renders the solid phase more stable than the supercooled liquid phase. In fact, the solid equilibrium globally minimizes the free-energy. In the absence of interfacial free-energy, the free-energy is $-|U| \operatorname{vol}\left(\Omega_{1}\right)$ and is hence lowered by any solution in which the solid phase grows in volume from its initial distribution with zero volume. Interfacial free-energy is stabilizing: in its presence the solid phase cannot grow in volume from zero volume and zero surface area; such solutions violate (2.4), since they have area (\lrcorner$(t)) / \operatorname{vol}\left(\Omega_{1}(t)\right)$ unbounded as $t \rightarrow 0^{+}$. On the other hand, it is possible to construct sets which grow from zero volume but nonzero surface area and which initially lower the freeenergy. Thus, granted a supercooled boundary, spontaneous growth of a solid seed of zero volume but suitable shape is to be expected. In the next section we will exhibit solutions which display this phenomenon.

3. The spherically symmetric problem with fast diffusion and without surface kinetics, with the liquid supercooled. The effects of capillarity are most easily discussed under the assumptions of isotropy, fast diffusion, negligible surface kinetics, and equal conductivities for the two phases:

$$
c_{1}=c_{2}=\beta=0, \quad k_{1}=k_{2} .
$$

As a further simplification, we restrict our attention to problems involving spherical symmetry with the underlying space $\mathbb{R}^{3}$, and therefore seek solutions of the form $u(r, t), \chi(r, t)$ with $r=|x|$. The partial differential equations (1.6) then reduce to

$$
u_{r r}+2 r^{-1} u_{r}=0 \text { in the solid and in the liquid, }
$$

while the free-boundary conditions (1.7) at a spherical surface of radius $\zeta(t)$ take the form

$$
u(\zeta(t), t)= \pm \alpha \zeta(t)^{-1}, \quad \zeta^{\prime}(t)=u_{r}\left(\zeta(t)_{1}, t\right)-u_{r}\left(\zeta(t)_{2}, t\right),
$$

where, for convenience, we have chosen a length scale with $k_{1} / l=k_{2} / l=1$. Here $\zeta(t)_{1}$ indicates the limit as $\zeta(t)$ is approached from the solid phase, and similarly 
for $\zeta(t)_{2}$, and the plus or minus sign is chosen according as the material is liquid or solid for $r$ close to $\zeta(t)$ with $r<\zeta(t)$. We will discuss solutions of (3.2), (3.3) for $\Omega$ all of $\mathbb{R}^{3}$ with

$$
u(r, t) \rightarrow U<0 \text { as } r \rightarrow \infty
$$

or for $\Omega$ the region exterior to a sphere with

$$
u_{r}=0 \quad \text { on } \partial \Omega, \quad u(r, t) \rightarrow U<0 \quad \text { as } r \rightarrow \infty,
$$

so that the inner spherical boundary is insulated. In addition, we will always have the exterior phase region (the phase region containing the point at infinity) liquid, so that, by (3.4) or (3.5), the liquid is supercooled for all sufficiently large $r$.

Remarks. (3a) The general solution of (3.2) is $B(t)+G(t) r^{-1}$; thus the temperature must be spatially constant in any phase region that contains the origin, or, when $\Omega$ is the region exterior to a sphere, in any phase region that contains the inner boundary. On the other hand, the temperature must have the form $U+G(t) r^{-1}$ in the exterior phase region.

(3b) Since $u_{t}(x, t)$ does not enter (3.2)-(3.5), solutions $u(x, t)$ need not be continuous in $t$, although the continuity of $\zeta(t)$ does impose some regularity.

3.1. Growth of the solid phase from a ball. Let $\Omega=\mathbb{R}^{3}$, and suppose that initially the solid occupies a ball of radius $R$, with the region exterior to $R$ liquid. Then, by (3.3) and Remark (3a), the interfacial radius $\zeta(t)$ satisfies the initial-value problem:

$$
\zeta^{\prime}=-(U \zeta+\alpha) / \zeta^{2}, \quad \zeta(0)=R
$$

Thus $U<0$ is necessary for the growth of the solid phase. Granted this,

$$
\begin{array}{lll}
\zeta(t) \rightarrow 0 \quad \text { in finite time } & \text { if } R<\alpha /|U| \\
\zeta(t) \rightarrow \infty \quad \text { as } t \rightarrow \infty & \text { if } R>\alpha /|U|, \\
\zeta(t) \equiv R & & \text { if } R=\alpha /|U|
\end{array}
$$

Therefore, for growth of a ball of the solid phase, it is necessary and sufficient that the liquid be supercooled and that the initial radius of the ball be larger than $\alpha /|U|$.

Let $R<\alpha /|U|$, and let $T$ denote the time at which the solid phase disappears. Then for $t>T$ the solution consists of the liquid equilibrium (2.9) (cf. Remark (3b)).

REMARK (3c). This discussion applies almost without change for $\Omega$ a ball of radius $R_{0}, u=U$ on $\partial \Omega$, and $R<R_{0}$, the only difference occurring when $R>\alpha /|U|$. In this case $\zeta(t) \rightarrow R_{0}$ in finite time $T_{0}$ (cf. $\left.(3.7)_{2}\right)$, so that for $t \geq T_{0}$ the solution is the solid equilibrium (2.10).

3.2. Growth from a set of arbitrarily small volume. Again let $\Omega=\mathbb{R}^{3}$. We choose an arbitrary point $\zeta_{0} \in(0,1)$ and seek a solution in which the solid phase is a spherical shell $\rho(t)<r<\zeta(t)$ with

$$
\rho(0)=\rho_{0}, \quad \zeta(0)=\rho_{0}+\varepsilon,
$$

with $\varepsilon>0$ small. In view of (3.3) and Remark (3a), finding such a solution reduces 


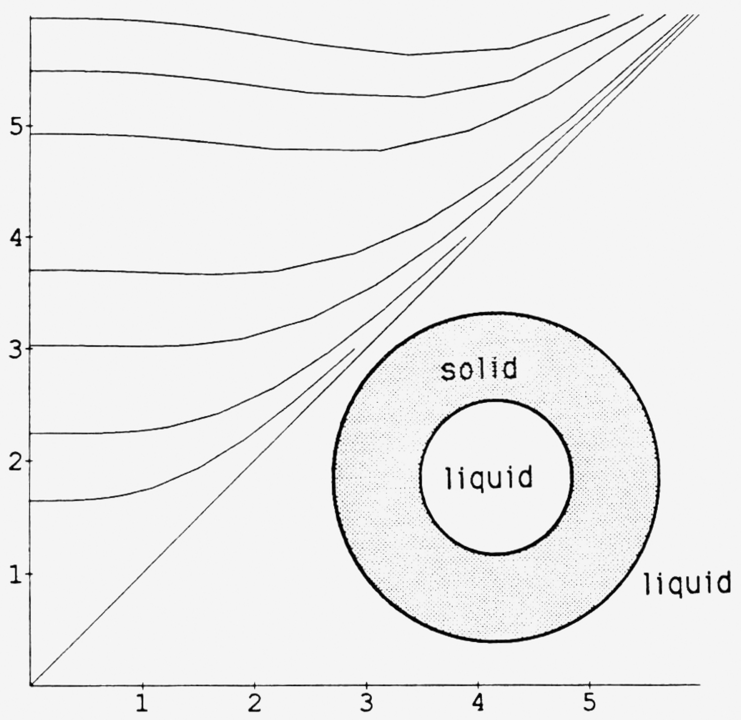

FIG. 1. The phase portrait for a solid shell of inner radius $\rho$ and outer radius $\zeta$ growing from the shell $\rho(0)=\rho_{0}, \zeta(0)=\rho_{0}+\varepsilon$. The inner ball of liquid disappears in finite time leaving a solid ball surrounded by liquid. This solid ball disappears in finite time or grows without bound according as $\varepsilon$ is less than or greater than $\varepsilon_{0}$.

to solving the differential equations

$$
\begin{aligned}
& \rho^{\prime}=-\alpha(\rho+\zeta) / \rho^{2}(\zeta-\rho), \\
& \zeta^{\prime}=-\{2 \alpha+U(\zeta-\rho)\} / \zeta(\zeta-\rho)
\end{aligned}
$$

subject to (3.8) and the constraint

$$
0<\rho(t)<\zeta(t) .
$$

Equations (3.9) are not defined at $\zeta=\rho$. In fact, we will show at the end of the section that there is no solution of (3.9), (3.10) for $t>0$ that satisfies the initial condition (3.8) with $\varepsilon=0$.

The phase diagram for (3.9) is shown in Fig. 1. The system (3.8)-(3.10) has a solution for each $\varepsilon>0$, and each such solution has $\rho(t) \rightarrow 0$ in a finite time $T(\varepsilon)$. At $t=T(\varepsilon)$ the inner liquid-phase disappears, and we are left with a solid of radius $R=\zeta(T(\varepsilon))$ surrounded by liquid. Thus for $t>T(\varepsilon)$ the solution is given by the solution of (3.6) with the initial condition applied at time $T(\varepsilon)$ (cf. Remark (3b)). Therefore, by (3.7) and Fig. 1, for $t>T(\varepsilon)$ the solid ball disappears in finite time $T^{*}(\varepsilon)$ or grows without bound according as $\varepsilon$ is less than or greater than a critical value. For $\varepsilon$ small, the solution for $t \geq T^{*}(\varepsilon)$ is the liquid equilibrium (2.9).

Consider the initial time-interval $t<T(\varepsilon)$. Letting $\delta=\zeta-\rho$, we may use (3.9) to conclude that

$$
\delta^{\prime}=\left\{\alpha\left(\zeta^{2}+\rho \zeta-2 \rho^{2}\right)-U \rho^{2} \delta\right\} /\left(\delta \zeta \rho^{2}\right),
$$

and therefore, even though $\zeta$ decreases, the distance $\delta$ between the interfaces increases. 
The limit $\varepsilon \rightarrow 0$ is interesting. We now show that, in this limit, the region occupied by the solid disappears infinitely fast; precisely,

$$
T^{*}(\varepsilon) \rightarrow 0 \quad \text { as } \varepsilon \rightarrow 0 .
$$

To prove (3.12), choose $\rho_{0}>0$ and $\varepsilon>0$, and let $\left(\rho_{\varepsilon}, \zeta_{\varepsilon}\right)$ denote the solution of $(3.8)-(3.10)$ on $[0, T(\varepsilon)]$, with $\zeta_{\varepsilon}$ extended continuously via $(3.6)_{1}$ to $\left[T(\varepsilon), T^{*}(\varepsilon)\right]$. Further, for $R \in\left(0, \rho_{0}\right)$, let

$$
T(\varepsilon, R)=\inf \left\{t \geq 0: \rho_{\varepsilon}(t)=R\right\}
$$

denote the time at which the inner radius reaches $R$. Our first step will be to show that for $\rho_{0}>0$ and $R \in\left(0, \rho_{0}\right)$ fixed,

$$
T(\varepsilon, R) \rightarrow 0 \text { as } \varepsilon \rightarrow 0 .
$$

Fix $\varepsilon>0$ and suppress the subscript $\varepsilon$. To verify (3.13), let $Q=\delta^{-1}$; then (3.9) has the form

$$
\begin{aligned}
& \rho^{\prime}=-\alpha(1+2 \rho Q) / \rho^{2}, \\
& Q^{\prime}=-Q^{2}\left\{\alpha(1+3 \rho Q)+|U| \rho^{2} Q\right\} / \rho^{2}(1+\rho Q) .
\end{aligned}
$$

Let $t$ be confined to the interval $[0, T(\varepsilon, R)]$. Then, since $(1+3 \rho Q) /(1+\rho Q) \leq 3$ for all $\rho Q \geq 0$, and since $\rho(t) \geq R$,

$$
Q^{\prime} \geq-C Q^{2}, \quad C=(3 \alpha+|U| R) / R^{2} .
$$

Further, $Q(0)=\varepsilon^{-1} ;$ thus

$$
Q(t) \geq(\varepsilon+C t)^{-1}
$$

On the other hand, $(3.14)_{1}$ yields

$$
\rho^{\prime}(t) \leq-2 \alpha Q(t) / \rho(t) \leq-2 \alpha /\left[\rho_{0}(\varepsilon+C t)\right] ;
$$

hence

$$
\rho(t) \leq \rho_{0}-\left(2 \alpha / \rho_{0} C\right) \ln [1+(C t / \varepsilon)] .
$$

Let $S(\varepsilon, R)$ denote the time at which the right side of this inequality reaches the value $R$. Then $S(\varepsilon, R) \rightarrow 0$ as $\varepsilon \rightarrow 0$. But $T(\varepsilon, R) \leq S(\varepsilon, R)$; thus (3.13) is satisfied.

Next, $(3.14)_{1}$ yields

$$
\left(\rho^{2}\right)^{\prime}=-4 \alpha Q-2 \alpha / \rho \geq-4 \alpha Q-2 \alpha / R .
$$

Thus, since $R=\rho(T(\varepsilon, R))$,

$$
R^{2} \geq \rho_{0}^{2}-(2 \alpha / R) T(\varepsilon, R)-4 \alpha \int_{0}^{T(\varepsilon, R)} Q(s) d s .
$$

Similarly, by $(3.9)_{2}$,

$$
\left(\zeta^{2}\right)^{\prime}=-4 \alpha Q+2|U|,
$$

so that

$$
\zeta^{2}(t)=\left(\rho_{0}+\varepsilon\right)^{2}-4 \alpha \int_{0}^{t} Q(s) d s+2|U| t
$$


Thus, by (3.16),

$$
\zeta^{2}(T(\varepsilon, R)) \leq\left(\rho_{0}+\varepsilon\right)^{2}-\rho_{0}^{2}+R^{2}+2 R^{-1}(\alpha+|U| R) T(\varepsilon, R),
$$

which allows us to choose $\varepsilon_{0}=\varepsilon_{0}(R)$ small enough that

$$
\zeta(T(\varepsilon, R)) \leq 2 R
$$

for $\varepsilon \in\left(0, \varepsilon_{0}\right)$.

We now consider times $t \in\left[T(\varepsilon, R), T^{*}(\varepsilon)\right]$. The function $\zeta(t)$ satisfies $(3.9)_{2}$ on $[T(\varepsilon, R), T(\varepsilon)]$ and $(3.6)_{1}$ on $\left[T(\varepsilon), T^{*}(\varepsilon)\right]$; thus

$$
\left(\zeta^{2}\right)^{\prime} \leq 2|U|-(2 \alpha / \zeta)
$$

on $\left[T(\varepsilon, R), T^{*}(\varepsilon)\right]$. We now require that $R$ be small enough that

$$
R \leq \alpha / 4|U|
$$

then, by (3.17) the right side of the above inequality is negative. Hence $\zeta$ is nonincreasing on $\left[T(\varepsilon, R), T^{*}(\varepsilon)\right]$ and, by $(3.17)$ and $(3.18),\left(\zeta^{2}\right)^{\prime} \leq-\alpha / 2 R$, so that

$$
\zeta^{2}(t) \leq 4 R^{2}-(\alpha / 2 R)[t-T(\varepsilon, R)]
$$

for $t \in\left[T(\varepsilon, R), T^{*}(\varepsilon)\right]$. Consequently

$$
T^{*}(\varepsilon) \leq T(\varepsilon, R)+8 R^{3} / \alpha .
$$

Letting $\varepsilon \rightarrow 0$ and then $R \rightarrow 0$, we arrive at (3.12).

We conclude this section by showing that the problem (3.8)-(3.10) with $\varepsilon=0$ has no solution. Suppose there were a solution. Choose a time $t_{0}>0$ and an $R \in\left(0, \rho_{0}\right)$ such that the solution exists on $\left[0, t_{0}\right]$ and $\rho(t) \in\left(R, \rho_{0}\right]$ on $\left[0, t_{0}\right]$. Then the steps leading to (3.15) here imply the existence of constants $C_{0}$ and $C$ such that

$$
Q(t) \geq\left(C_{0}+C t\right)^{-1} \text {. }
$$

Further, since $\delta(0)=0$, we must have $C_{0}=0$. Thus $(3.14)_{1}$ yields

$$
\rho^{\prime}(t) \leq-2 \alpha Q(t) / \rho(t) \leq-2 \alpha / \rho_{0} C t,
$$

and, for $t \in\left(0, t_{0}\right)$,

$$
\rho\left(t_{0}\right) \leq \rho(t)-\left(2 \alpha / \rho_{0} C\right) \ln \left(t_{0} / t\right) .
$$

Letting $t \rightarrow 0$ yields a contradiction, since $\rho\left(0^{+}\right)=\rho_{0}$. Thus the problem with $\varepsilon=0$ has no solution.

3.3. Region exterior to a sphere with inner boundary insulated: growth from a set of zero volume. This problem furnishes an example of growth from a set of zero volume. Here $\Omega$ is the region in $\mathbb{R}^{3}$ exterior to a sphere of radius $R$, and the "boundary conditions" are (3.5), so that the inner boundary is insulated, while the boundary at infinity is supercooled. We seek a solution in which the solid phase is a spherical shell $R<r<\zeta(t)$ for $t>0$ with

$$
\zeta(0)=R,
$$

so that initially the solid phase has zero volume. 
In view of Remark (3a), the current problem-(3.2), (3.3), (3.5), and (3.19)leads to (3.6), and we may therefore conclude that for $R>\alpha /|U|$ there is a solution in which the solid phase grows without bound. For $R<\alpha /|U|$ there is no solution with the solid phase a spherical shell $R<r<\zeta(t)$ for $t>0$; for this range of $R$ the solid phase disappears immediately, and the solution is the liquid equilibrium (2.9). For $R=\alpha /|U|$ there are two possibilities: the liquid equilibrium; the formal solution in which the solid phase remains in a thin spherical shell of zero thickness at $r=R$. The second solution has larger free-energy (2.3) than the first, since the solid-liquid interface has interfacial free-energy (2.5).

3.4. Some general remarks. In the problems of the last three subsections, the volume $\operatorname{vol}\left(\Omega_{1}(t)\right)$ of the solid phase is continuous, and so is the interfacial area, area $(s(t))$, except when the interface reaches the boundary, as in Remark (3c). (We define area $(s(t))=0$ for the solid and liquid equilibria.)

The problem discussed in Sec. 3.3 consists in solving (3.2)-(3.4) for $t>0$ in conjunction with the initial condition: $\chi(r, 0)=1, r \neq \zeta_{0}, \chi\left(\zeta_{0}, 0\right)=0$, a condition which represents an infinitesimally thin spherical shell of the solid phase situated at $r=\zeta_{0}$, so that, formally, area $(\diamond(0)) \neq 0$. There are at least two solutions of this problem: the solution given in Sec. 3.2 and the liquid equilibrium (2.10). For the latter, area $(s(t))$ is discontinuous at $t=0$, but area $(s(t))$ is discontinuous also in the solution discussed in Remark (3c). In addition, the liquid equilibrium lowers the free-energy as the two interfaces at $r=\zeta_{0}$ disappear.

Given a solution, a time $t_{0}$, and a radius $\zeta_{0}$ in the liquid region at $t_{0}$, one might ask whether it is reasonable to consider a second solution in which a solid region of zero thickness at $r=\zeta_{0}$ forms spontaneously at $t_{0}$. Such a solution is not acceptable physically, since it raises the total free-energy at time $t_{0}$ by an amount equal to the interfacial free-energy of the two interfaces at $r=\zeta_{0}$. In fact, a physical requirement for any solution is that area $(s(t))$ not suffer a positive jump discontinuity.

3.5 Reduction to a Stefan problem. Ostensibly, one expects the problem with capillarity to be more stable than the Stefan problem, but, interestingly, the former may be reduced to the latter by a change in dependent variable, at least when the interface consists of a single spherical surface of radius $\zeta(t)$.

Assume first that $\Omega$ does not contain the origin and define a new dependent variable

$$
w(r, t)=u(r, t) \pm \alpha r^{-1},
$$

where the plus or minus sign is chosen according as the material is solid or liquid for $r$ close to $\zeta(t)$ with $r<\zeta(t)$. Then $w(r, t)$ satisfies

$$
w_{r r}+2 r^{-1} w_{r}=0 \text { in the solid and in the liquid, }
$$

and

$$
w(\zeta(t), t)=0, \quad \zeta^{\prime}(t)=u_{r}\left(\zeta(t)_{1}, t\right)-u_{r}\left(\zeta(t)_{2}, t\right),
$$

which are exactly the free-boundary conditions of the Stefan theory.

When $\Omega$ contains the origin, we replace $(4.15)$ by

$$
w(r, t)=u(r, t) \pm f(r),
$$


where $f(r)$ is smooth for all $r$ and equal to $\alpha r^{-1}$ outside a ball $\Gamma$ of sufficiently small radius. Then-provided the interface lies outside of $\Gamma$-the free-boundary conditions (3.22) remain unchanged; on the other hand, the differential equation (3.21) will have a nonzero right-hand side within $\Gamma$.

The transformations (3.20) and (3.23) apply also to the problem without the assumption of fast diffusion, in which case (3.22) remains unchanged, while (3.21) has, on the right-hand side, the term $c_{1} u_{t}$ in the solid and the term $c_{2} u_{t}$ in the liquid.

4. A weak formulation of the general problem. The general problem associated with (1.2), (1.3) is extremely difficult, ${ }^{11}$ chiefly because of the presence of curvature and normal velocity in the free-boundary condition. In this section we suggest a possible weak formulation, assuming, for convenience, that $\Omega=\mathbb{R}^{d}$ to avoid discussing boundary conditions.

Guided by the physics, we divide the basic equations into two parts:

(i) the energy equation:

$$
\begin{array}{cl}
c_{1} u_{t}=-\operatorname{div} \mathbf{q}, & \mathbf{q}=-\mathbf{K}_{1} \nabla u \text { in the solid, } \\
c_{2} u_{t}=-\operatorname{div} \mathbf{q}, & \mathbf{q}=-\mathbf{K}_{2} \nabla u \text { in the liquid, } \\
l v=[\mathbf{q}] \cdot \mathbf{n} & \text { on the interface; }
\end{array}
$$

(ii) the interface condition:

$$
u=\mathbb{B}(\mathbf{n}) \cdot \mathbb{L}-\beta(\mathbf{n}) v \quad \text { on the interface. }
$$

The energy equations (4.1) are consequences of balance of energy and the bulk constitutive equations, the jump condition (4.1b) arising because of constitutive differences between the bulk phases; the interface condition (4.2) is a consequence of the constitutive equations for the interface in conjunction with thermodynamic arguments (cf. [10]).

In the classical Stefan problem, the solid and liquid are defined by $u<0$ and $u>0$, respectively, and the bulk energy is given by $c_{1} u$ in the solid and $l+c_{2} u$ in the liquid. In fact, defining a global energy $e(u)$ and a global heat flux $\mathbf{q}(\nabla u)$ by

$$
\begin{aligned}
& e(u)=c_{1} u+h(u)\left[l+\left(c_{2}-c_{1}\right) u\right], \\
& \mathbf{q}(\nabla u)=-\left[\mathbf{K}_{1} \nabla u+h(u)\left(\mathbf{K}_{2}-\mathbf{K}_{1}\right) \nabla u\right],
\end{aligned}
$$

where $h$ is the heaviside function, the energy equations (4.1) are formally equivalent to the single equation

$$
e(u)_{t}=-\operatorname{div} \mathbf{q}(\nabla u)
$$

to be interpreted in the sense of distributions. The standard weak formulation of the classical Stefan problem is based on (4.4).

The presence of supercooling and/or superheating-which is necessary if the temperature at the interface is to satisfy (4.2) rather than $u=0$-render (4.3) inapplicable. In this case, motivated by (4.3), we use the phase distribution $\chi$ to define the

\footnotetext{
${ }^{11}$ Luckhaus [16] establishes global existence and lack of uniqueness for certain weak solutions of the isotropic problem with equal heat capacities. Duchon and Robert [7] establish local existence and uniqueness for the isotropic one-phase problem with fast diffusion.
} 
global energy $e(u, \chi)$ and the global heat flux $\mathbf{q}(\nabla u, \chi)$ through

$$
\begin{aligned}
& e(u, \chi)=c_{1} u+\chi\left[l+\left(c_{2}-c_{1}\right) u\right], \\
& \mathbf{q}(\nabla u, \chi)=-\left[\mathbf{K}_{1} \nabla u+\chi\left(\mathbf{K}_{2}-\mathbf{K}_{1}\right) \nabla u\right]
\end{aligned}
$$

and this formally reduces the energy equations to the distributional equation

$$
e(u, \chi)_{t}=-\operatorname{div} \mathbf{q}(\nabla u, \chi) .
$$

Our next step is to write a weak form for the interface condition. Here we use an idea due to Sethian [9] and Osher and Sethian [11], who note that equations of the form (4.2) (with $u$ interpreted as data) arise automatically as equations satisfied by the level surface of solutions of certain partial differential equations defined on all of $\mathbb{R}^{3}$.

To apply the Osher-Sethian procedure to (4.2), we define $b(\mathbf{v})$ and $\mathbf{B}(\mathbf{v}) \in \operatorname{lin}\left(\mathbb{R}^{3}\right)$ for any vector $\mathbf{v} \neq \mathbf{0}$ by

$$
b(\mathbf{v})=\beta(\mathbf{w}), \quad \mathbf{B}(\mathbf{v})=\mathbb{I}(\mathbf{w}) \mathbb{B}(\mathbf{w}) \mathbb{P}(\mathbf{w}), \quad \mathbf{w}=|\mathbf{v}|^{-1} \mathbf{v},
$$

where $\mathbb{P}(\mathbf{w})$ is the projection of $\mathbb{R}^{3}$ onto $\mathbf{w}^{\perp}$, while $\mathbb{I}(\mathbf{w})$ is the inclusion of $\mathbf{w}^{\perp}$ into $\mathbb{R}^{3}$. Next, we note that, given a function $\varphi$

$$
\mathbf{n}=\nabla \varphi /|\nabla \varphi|, \quad v=-\varphi_{t} /|\nabla \varphi|
$$

give the unit normal and normal velocity of level surfaces of $\varphi$, with $\mathbf{n}$ pointing in the direction of increasing $\varphi$, and further

$$
\mathbb{L}=|\nabla \varphi|^{-1} \mathbb{P}(\mathbf{n}) \nabla \nabla \varphi \mathbb{I}(\mathbf{n})
$$

is the corresponding curvature tensor. Thus, formally, the level surfaces of any function $\varphi(x, t)$ consistent with the equation

$$
b(\nabla \varphi) \varphi_{t}=\mathbf{B}(\nabla \varphi) \cdot \nabla \nabla \varphi+|\nabla \varphi| u
$$

will evolve according to the interface condition (4.2). This allows us to replace the interface condition (4.2) by (4.10), with the interface the zero-level surface of $\varphi$ and the region occupied by the solid the set on which $\varphi<0$. An advantage of considering the interface in this manner is that (4.10) admits a weak interpretation in the viscosity sense; ${ }^{12}$ that is, as a viscosity solution in the sense of Crandall and Lions [6].

We are therefore led to formally consider the following system ${ }^{13}$ for $u(x, t)$ and $\chi(x, t)$ :

$$
\begin{aligned}
& e(u, \chi)_{t}=-\operatorname{div} \mathbf{q}(\nabla u, \chi) \quad \text { in the sense of distributions, } \\
& b(\nabla \varphi) \varphi_{t}=\mathbf{B}(\nabla \varphi) \cdot \nabla \nabla \varphi+|\nabla \varphi| u \text { in the viscosity sense }
\end{aligned}
$$

\footnotetext{
${ }^{12} \mathrm{Cf}$. Chen, Giga, and Goto [13], Evans and Spruck [14], and Soner [17], who use viscosity solution of equations of the form (4.12) to study surfaces that evolve according to equations of the form (4.2) ( $u$ prescribed).

${ }^{13}$ This formulation was discovered independently by Sethian and Strain and presented by Strain at the SIAM meeting, Chicago, July 1990. The formulation was also noted independently by Noel Walkington (private communication).
} 
with $e(u, \chi)$ and $\mathbf{q}(\nabla u, \chi)$ defined by (4.5) and

$$
\chi(x, t)= \begin{cases}0 & \text { whenever } \varphi(x, t)<0 \\ 1 & \text { whenever } \varphi(x, t)>0 .\end{cases}
$$

Acknowledgment. We gratefully acknowledge the Army Research Office and the National Science Foundation for their support of this research.

\section{REFERENCES}

[1] W. W. Mullins, Two-dimensional motion of idealized grain boundaries, J. Appl. Phys. 27, 900-904 (1958)

[2] P. G. Saffman and G. I. Taylor, The penetration of a fluid into porous medium or Hele-Shaw cell containing a more viscous liquid, Proc. Roy. Soc. London Ser. A 245, 312-329 (1958)

[3] W. W. Mullins, Grain boundary grooving by volume diffusion, Trans. Met. Soc. AIME 218, 354-361 (1960)

[4] W. W. Mullins and R. F. Sekerka, Morphological stability of a particle growing by diffusion or heat flow, J. Appl. Phys. 34, 323-329 (1963)

[5] J. W. McLean and P. G. Saffman, The effect of surface tension on the shape of fingers in a Hele-Shaw cell, J. Fluid. Mech. 102, 455-469 (1981)

[6] M. G. Crandall and P. L. Lions, Viscosity solutions of Hamilton-Jacobi equations, Trans. Amer. Math. Soc. 277, 1-42 (1983)

[7] J. Duchon and R. Robert, Evolution d'une interface par capillarité et diffusion de volume, existence local en temps, C. R. Acad. Sci. Paris Sér. I Math. 298, 473-476 (1984)

[8] G. Barles, Remark on a flame propagation model, Rapport INRIA, No. 464, 1985

[9] J. A. Sethian, Curvature and the evolution of fronts, Comm. Math. Phys. 101, 487-499 (1985)

[10] M. E. Gurtin, Multiphase thermomechanics with interfacial structure. 1. Heat conduction and the capillary balance law, Arch. Rational Mech. Anal. 104, 195-221 (1988)

[11] S. Osher and J. A. Sethian, Front propagation with curvature dependent speed: Algorithms based on Hamilton-Jacobi formulations, J. Comput. Phys. 79, 12-49 (1988)

[12] S. Angenent and M. E. Gurtin, Multiphase thermomechanics with interface structure. 2. Evolution of an isothermal interface, Arch. Rational Mech. Anal. 108, 323-391 (1989)

[13] Y. -G. Chen, Y. Giga, and S. Goto, Uniqueness and existence of viscosity solutions of generalized mean curvature flow equations, J. Diff. Geometry, to appear

[14] L. C. Evans and J. Spruck, Motion of level sets by mean curvature, I, J. Diff. Geometry, II, Trans. Amer. Math. Soc., to appear

[15] M. E. Gurtin , G. Kossioris, and H. M. Soner, The one-dimensional superthermal Stefan problem, forthcoming

[16] S. Luckhaus, Solutions for the two-phase Stefan problem with the Gibbs-Thomson law for the melting temperature, European J. Appl. Math. 1, 101-111 (1990)

[17] H. M. Soner, Motion of a set by the curvature of its boundary, J. Differential Equations, to appear

[18] E. Yokoyama and T. Kuroda, Pattern formation in growth of snow crystals occurring in the surface kinetic process and the diffusion process, Phys. Rev. A(3) 41, 2038-2049 (1990) 